\title{
Reconstruction of Orbital Walls With 3D Printed Patient Specific Implant Using PEEK: A Case Report
}

\author{
Ali Mahfuri ${ }^{1}$, Kenan Saoud ${ }^{1 *}$, Yasser Al-moudallal ${ }^{1}$ and Muaaz Alkhouli ${ }^{2}$ \\ ${ }^{1}$ Department of oral and maxillofacial surgery, Faculty of Dentistry, Damascus University, Syria
}

${ }^{2}$ Department of Pediatric Dentistry, Faculty of Dentistry, Damascus University, Syria

*Corresponding author: Kenan Saoud, Department of Oral \& Maxillofacial surgery, Faculty of dentistry, Damascus University, Al-Mazzeh-St. Syria, zip code: 96311, Syria

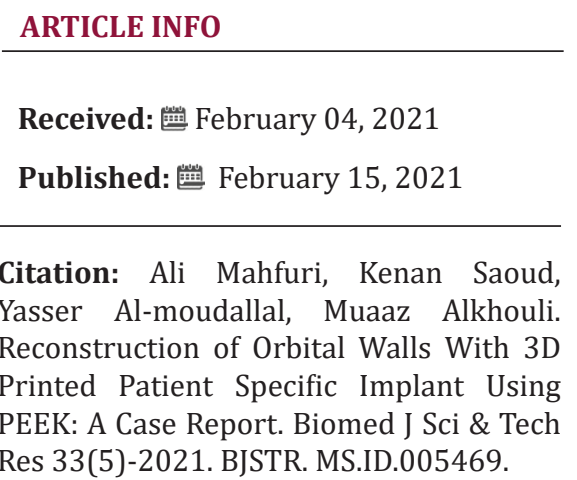

\section{ABSTRACT}

Cones beam conventional tomography (CBCT), virtual studies and 3D printing are of great importance in the field of oral and maxillofacial surgery as well in the restoration of defects resulting from various injuries. The combination of the three previous aspects led to production of custom implants for the reconstruction of craniofacial defects. These implants gave positive results in the maxillofacial surgery in terms of reducing the surgical time, the precise adaptation to the region of implantation and better cosmetics. In addition to the variety of biocompatible materials that could be used in the manufacture of these implants, each material possesses distinct properties that justify its use in restoring craniofacial defects.

Keywords: 3D Printing; PEEK; CBCT; Reconstruction; Virtual Studies; Patient Specific Implant (PSI), Orbit

\section{Introduction}

Head injuries pose a significant challenge to physicians due to their being of high risk for patients. One type of them is maxillofacial injuries that cause both functional and esthetic consequences that are considered difficult to manage [1]. The process of reconstructing orbital walls is a challenging procedure due to the complexity of the anatomical area and its great esthetic importance [2]. Zygomatic bone is one of the bones contributing to the formation of the orbital walls [3]. It is significantly vulnerable to fracture, and it can be considered the second most common facial bones fracture after fractures of the nasal bones [4]. Diagnosis of zygomaticomaxillary complex fractures is based on clinical and radiographic examination. CT scan is the best radiographic method in diagnosing such fractures especially in the assessment of orbital floor and walls $[4,5]$.

Different types of grafting materials are used to reconstruct maxillofacial defects; they are divided into four main categories: autografts, allografts, xenografts and alloplastic (synthetic) grafts [6]. Autologous bone grafts remain the gold standard for reconstruction of bony defects during the management of maxillofacial defects [7]. However, harvesting bone from the donor site could be opposed by some limitations and complications like additional surgery and operative time, donor site morbidity, the amount of bone needed to harvest from the donor site, pain in the donor site, bone graft resorption and difficulty with contouring [7-9].

One of the alloplastic materials that could be used is polyetheretherketon (PEEK), which is a semicrystalline polyaromatic polymer that combines durability, stiffness, and biocompatibility. Due to its beneficial characteristics, PEEK has been used in different medical applications [10]. Most distinctly, PEEK has shown an evidence-based success in managing disk diseases of the cervical vertebrae. In addition, PEEK has been constituted as a surrogate for autogenous bone grafts in different vertebral problems [11-13]. Moreover, in order to achieve an accurate restoration of the defect, the dimensions should be measured with an accurate method. CBCT images have been used in the literature to provide a precise measurement of these dimensions [14]. On the other hand, $3 \mathrm{D}$ printing has received great attention in the medical field due to the multiplicity of the domains that can be used for, ease of use, accuracy of prostheses manufactured with it, and the possibility of using a large number of biocompatible materials such as polymers, ceramics, metals, and composites [15]. Case Reports of the use of PEEK in the repair of maxillofacial defects have been insufficient 
[16]. However, we admit that PEEK coupled with a 3D-printing process that can produce custom-made implants can represent an optimal approach in the reconstruction of such challenging defects.

\section{Case Report}

A male patient of 18 years old came to the oral and maxillofacial surgery department in the faculty of dentistry at Damascus University with an injury in the right side of the face, the patient was referred from an ophthalmologist, who requested the reconstruction and restoration of the fractured orbital walls so that he could place an artificial eyeball. By clinical examination of the patient, it is evident that the ball of the right eye is lost due to injury, atelectasis in the right orbit of the walls, and asymmetry with the left (Figure 1). By reviewing the patient's medical history and studying the attached radiographs, the fracture has been treated previously using a titanium mesh in the right parietal bone. However, the lateral and inferior walls of the orbit have not been treated on the right side as well (Figure 2). After studying the case, it was found out that restoration should be made to restore for the material loss in the orbital walls and floor, PEEK was chosen as a replacement material. A CBCT image of the skull was performed using (Vatech PAX-i3D GREEN ,13, Samsung, Gyeonggido,445-170, Korea) to use in the prosthesis fabrication (Figure 3). The restoration was designed using the Exocad® (Exocad GmbH, Germany, 2010) software, in which a visual transfer of the healthy side was performed and represented on the affected side (Figure 4). Restoration fabrication was done using a 3D printer based on PEEK molds (Figure 5).

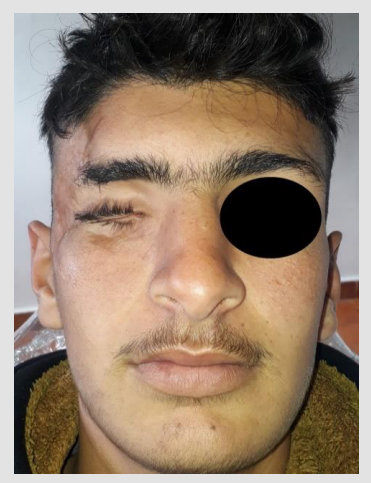

Figure 1: Absence of the right eyeball with atelectasis in the lateral wall of the right orbit.

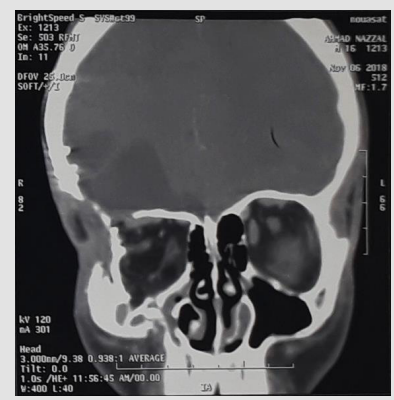

Figure 2: fractures in the lateral and lower and floor of the orbit.

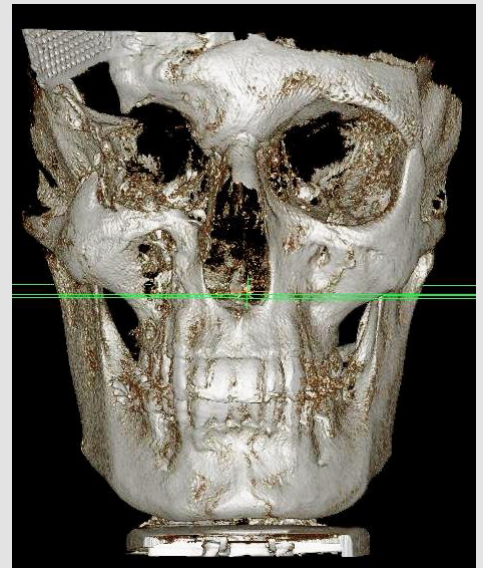

Figure 3: A three-dimensional view of the radiograph of the skull.

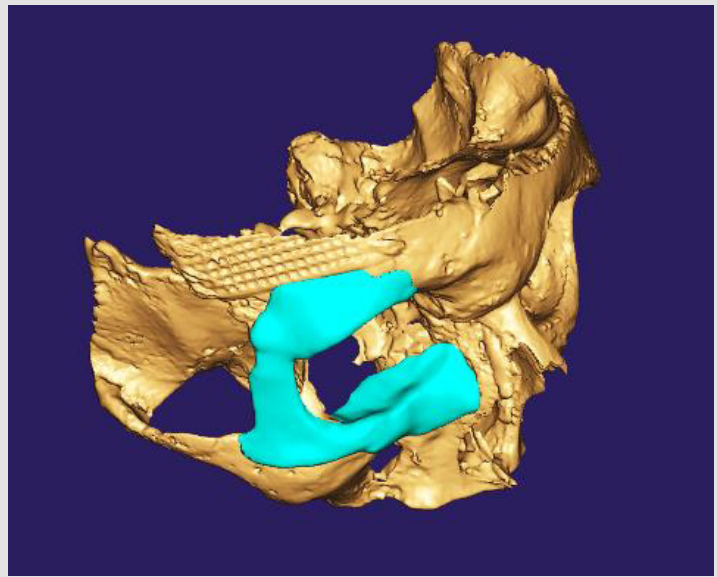

Figure 4: Representation of the restoration of the affected side virtually.

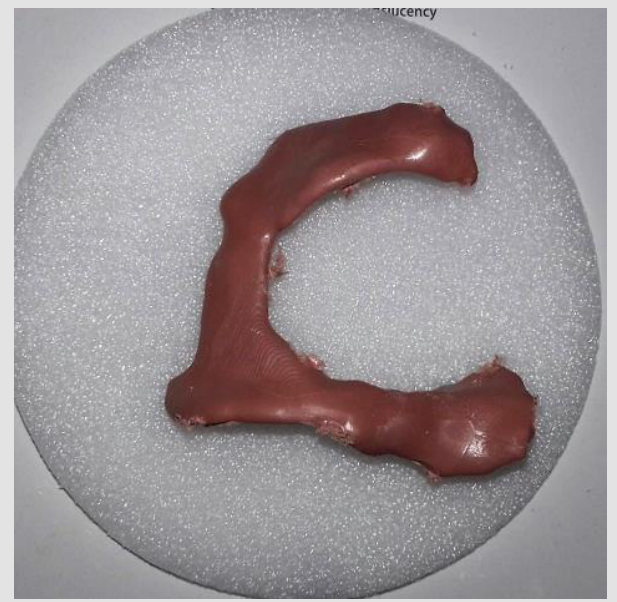

Figure 5: The restoration after printing and before finishing and polishing.

\section{Surgical Procedure Steps}

The patient was prepared for surgery after performing all the consultations and medical laboratory analysis necessary to undergo 
the surgery under general anesthesia. In the surgical portal, the coronal approach was performed; the patient's previous surgical scar was used (Figure 6). Then, the periosteum was elevated in the area closest to the work area to expose the area of bone loss (Figure 7). The upper portion of the prosthesis was then fixed with a screw after splitting the printed restoration into two pieces to facilitate its access and fixation and to make it easier to handle (Figure 8). Subtarsal approach to enable the lower part of the prosthesis to be screwed (Figure 9). Suture of the incisions was done by layers using Vicryl 3/0 for the suturing of muscles and periosteum, 3/0 Nylon sutures were used for skin closure. A compressive dressing was applied for 24 hours postoperatively with the necessary prescription and postoperative instructions were given. Photo at the follow-up 14 days after surgery, noting the return of the periorbital contour with a little edema remains (Figure 10), and a photo after 4 months (Figure 11). By reviewing the patient's medical history and studying the attached radiographs, a fracture treated using a titanium mesh in the right parietal bone and a fracture in the lateral and lower wall of the orbit and the floor of the orbit on the right side as well (Figure 2). As a result of the case study, it was found out that restoration should be made to restore for the material loss in the orbital walls and floor, and PEEK was chosen as a replacement material.

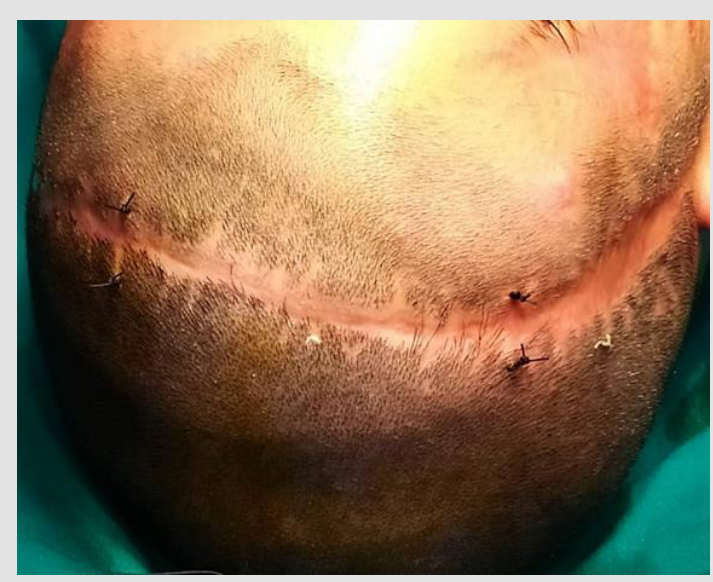

Figure 6: coronal approach.

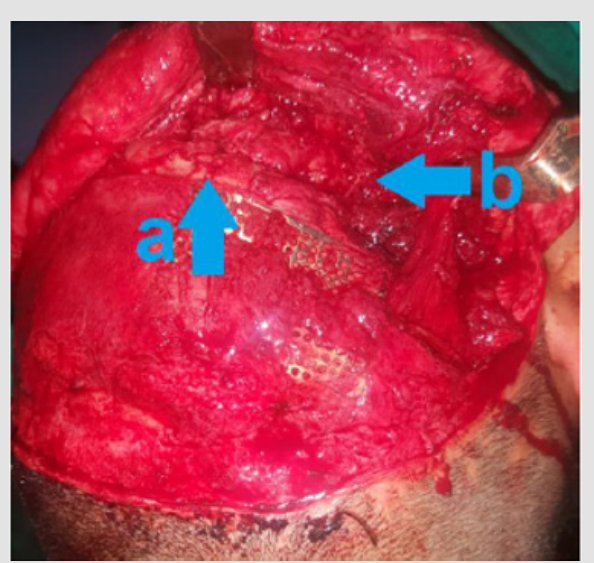

Figure 7: A- elevation of periosteum, b- bone loss area.

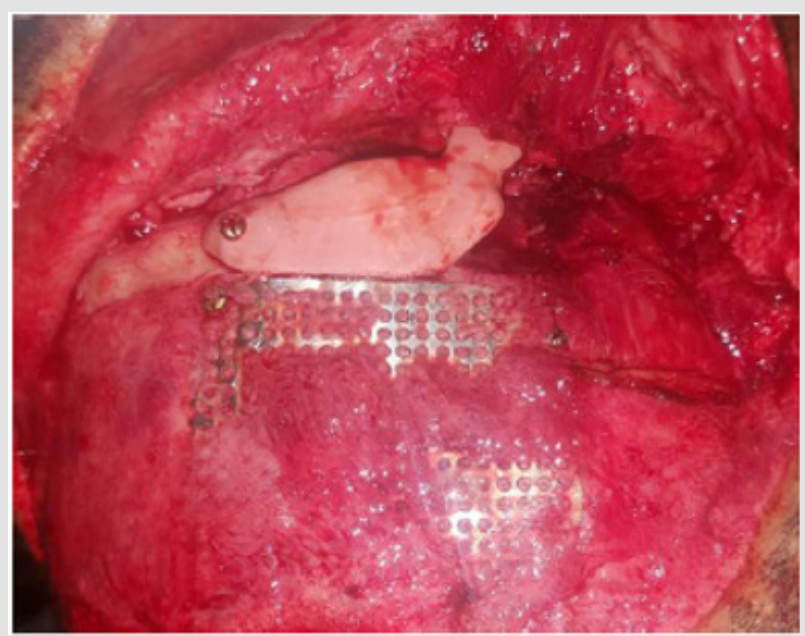

Figure 8.

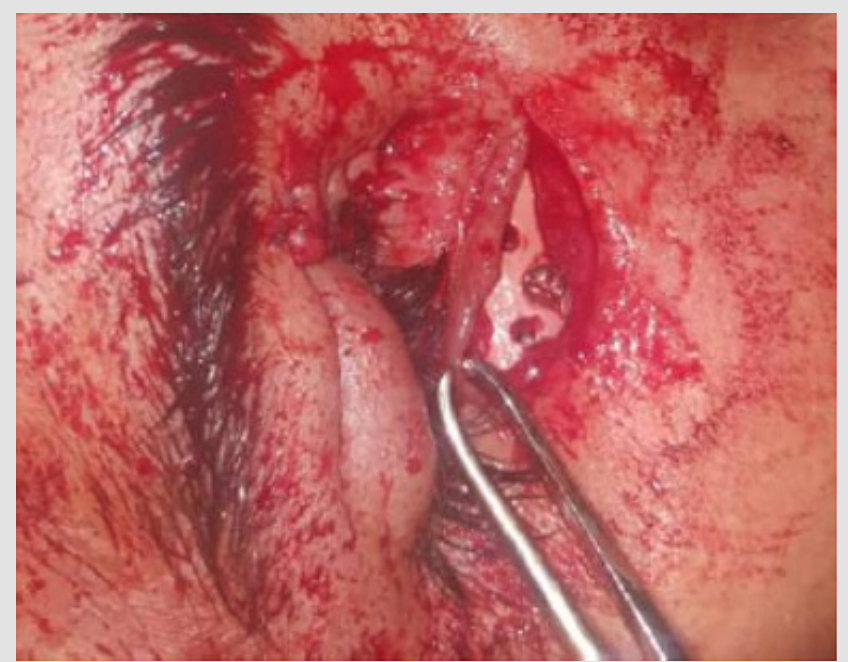

Figure 9.

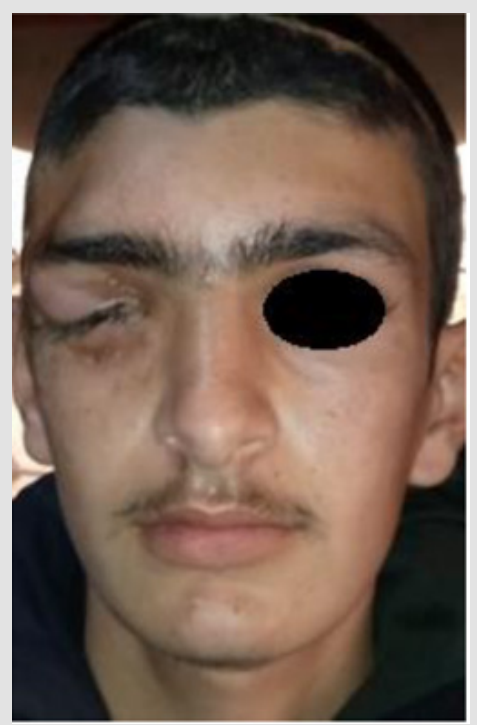

Figure 10. 

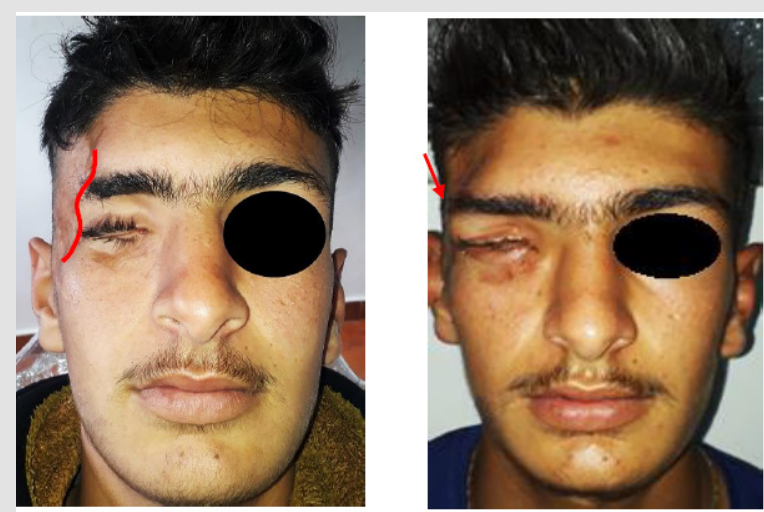

Figure 11: Comparison between the primary situation and 4 months after.

A CBCT image of the skull was performed using (Vatech PAXi3D GREEN ,13, Samsung, Gyeonggi-do,445-170, Korea) for use in the prosthesis fabrication (Figure 3). The restoration was designed using the Exocad ${ }^{\circledR}$ (Exocad GmbH, Germany, 2010) software, in which a visual transfer of the healthy side was performed and represented on the affected side (Figure 4). Restoration fabrication was done using a 3D printer based on PEEK molds (Figure 5). Dissection of the flap of the periosteum (Figures 12 \& 13). Elevating the periosteum in the area closest to the work area and exposing the area of bone loss (Figure 9), and fixation of the upper portion of the prosthesis with screw (Figure 10). Subtarsal approach to enable the lower part of the prosthesis to be screwed (Figure 11). Suture of the incisions were done on layers using Vicryl 3/0 for the suturing of muscles and periosteum, 3/0 Nylon sutures were used for skin closure. A compressive dressing was applied for 24 hours postoperatively with the necessary prescription and postoperative instructions given Photo at the follow up 14 days after of surgery, noting the return of the periorbital contour with a little edema remains (Figure 12), and a photo after 4 months (Figure 13).

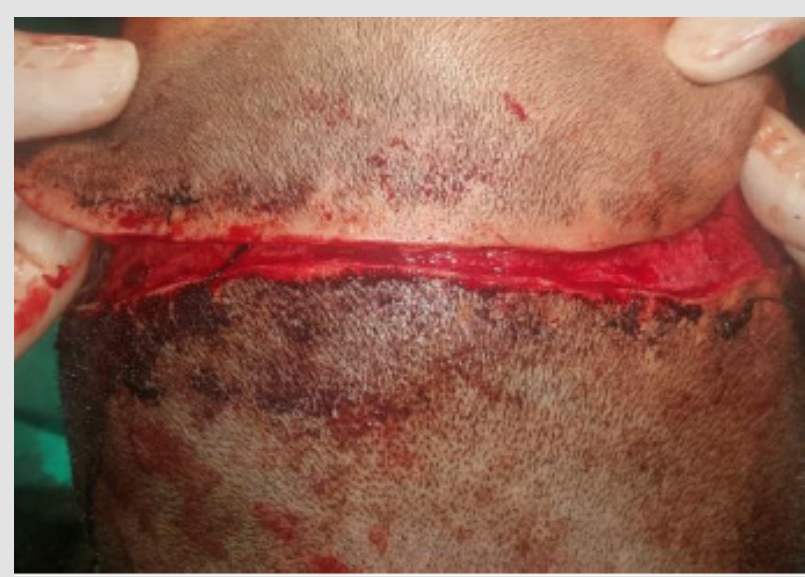

Figure 12.

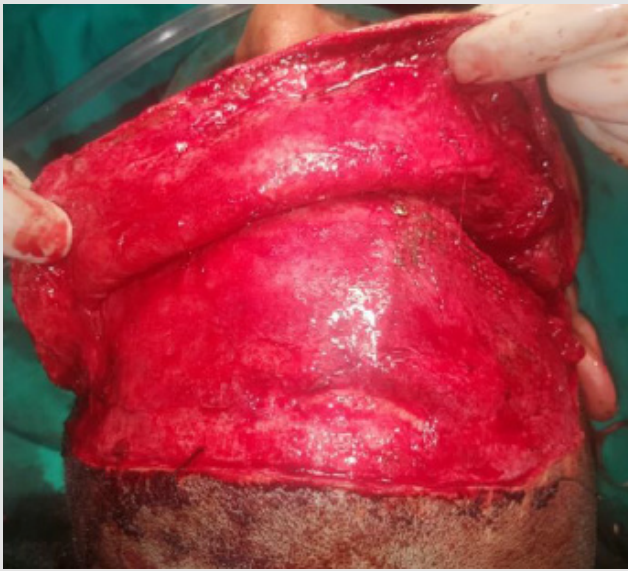

Figure 13.

\section{Discussion}

Maxillofacial defects can result from different conditions, including traumatic injuries and after resection of neoplastic lesions. The resultant defects are complicated as different types of tissues are lost which affects both functional and esthetic domains. For that reason, surgical practitioners have moved to various autogenous and alloplastic materials to rebuild the defects. All materials exhibit many drawbacks, as it is accepted that "there is no optimal restoration material for maxillofacial defects." To avoid the complications that may occur when using autografts, the use of bone substitutes and alloplastic materials has been used more. the most alloplastic materials used in oral and maxillofacial reconstruction are iron, titanium, hydroxyapatite, chrome-cobalt, methyl methacrylate, and other polymers [17]. One of the most widely used polymers in the medical field is Polyetheretherketon (PEEK), it is a polyaromatic semi-crystalline thermoplastic polymer with mechanical properties appropriate for medical applications like spine surgery, orthopedic surgery, maxillofacial surgery, cardiac surgery and dental applications, etc. PEEK has high chemical resistance, radiolucent, mechanical characteristics similar to bone, sterilizable and can be shaped and contoured by machine, all these properties made PEEK good alternative for metallic implants [18].

By using this material, we aimed to avoid the patient to undertake surgery in a second place such as iliac crest as the donor site and used the newly available techniques to design and manufacture the protheses by 3D printer with achieving satisfying results for the patient and the surgical team as well. Moreover, for the surgical portal in this case we depend on the presence of the scare resulted from the previous surgery to do our incision without making a scare in another place. During the surgery we found that the PEEK material is easy to handle and can be modified by using the bur with some saline irrigation when it is needed to quarantine the 
good outer shape and the symmetry with the intact side. Recently, the use of PEEK to reconstruct defects of the maxillofacial facial region has become widespread, especially with the development of 3D printing and its increased accuracy [19]. The great challenges facing traditional reconstruction of maxillofacial defects have imposed a trend towards 3D printing technology, this technology has provided physicians with the ability to manufacture patient specific prosthesis to rehabilitate cranio-maxillofacial defects, the sites where 3D printing restoration techniques are most commonly used in the cranium are oral, orbital, nasal and auricular regions [20]. The development of 3D printing has greatly facilitated the fabrication of the oral and maxillofacial prosthesis in a method of rapid prototyping [14], 3D printing depends on different types of radiographs such as CT, MRI, and CBCT, which are high-resolution imaging methods $[14,21]$.

In recent years, there has been a great development in the method of imaging using the CBCT, as these imaging devices are characterized by a low amount of irradiation, a high-resolution X-ray. For many of the advantages of CBCT imaging, it has been used extensively in reconstructive maxillofacial defects and virtual studies of various types of surgeries to plan for them and evaluating their results, in addition to print the anatomical structures, which facilitated surgery and reduced possible complications [21]. The results achieved in this case were very accurate in terms of the conformity of compensation to the computer-aided design and the applicability of it and are encouraging to use in similar cases.

\section{Conclusion}

In the future, dependence on 3D radiographs, 3D printing and virtual studies in the repair of maxillofacial defects will increase due to its great advantages in terms of facilitating surgery, reducing the time of surgery, the ability to predict the outcome with the distinctive results that it gives after surgery.

\section{Conflict of Interest}

The authors declare no conflict of interests with this case report.

\section{References}

1. J Parthasarathy (2014) 3D modeling, custom implants, and its future perspectives in craniofacial surgery. Annals of Maxillofacial Surgery 4(1): 9-18.

2. Klaus Sinko, Guido Dorner, Arnulf Baumann (2015) Late Reconstruction of the Orbit with Patient-Specific Implants Using Computer-Aided Planning and Navigation. J Oral Maxillofac Surg 73(12 Suppl): 101-106.

3. Margaret J Fehrenbach (2012) Skeletal System. Illustrated anatomy of the head and neck ( $4^{\text {th }}$ Edn.). St. Louis, Elseveir saunders, Missouri, USA pp. 31-85.
4. Raymond J Fonseca (2013) Fractures of the Zygomatic Complex and Arch. Oral and maxillofacial trauma ( $4^{\text {th }}$ Edn.). St. Louis, Elseveir Saunders, Missouri, USA, pp. 377-438.

5. Juan Marcelo Reyes, María Fernanda García Vargas, Jonathan Rosenvasser, María Asunción Arocena (2013) Classification and epidemiology of orbital fractures diagnosed by computed tomography. Revista Argentina de Radiología pp. 139-146.

6. Luciano Vidal, Carina Kampleitner, Meadhbh Á Brennan, Alain Hoornaert (2020) Reconstruction of Large Skeletal Defects: Current Clinical Therapeutic Strategies and Future Directions Using 3D Printing. Frontiers in Bioengineering and Biotechnology.

7. Arin K Greene, Gary F Rogers (2012) Autogenous Bone Graft: Basic Science and Clinical Implications," The Journal of Craniofacial Surgery 23(1): 323-327.

8. Neha Goel, Franco De Monte, Matthew M Hanasono (2012) Calvarial Reconstruction with Polyetheretherketone Implants. Annals of Plastic Surgery 23(1): 653-655.

9. Kavosh Zandsalimi, Morteza Rasoulianboroujeni, Lobat Tayebi, Reza Masaeli (2018) Challenges in 3D Printing of Bone Substitutes. Mary Ann Liebert 25(1).

10. Irfan Nawaz, Zhi Yang Ng (2014) Computer-Designed PEEK Implants: A Peek into The Future of Cranioplasty? The Journal of Craniofacial Surgery 25(1): 55-58.

11. G Michelinakis (2017) The use of cone beams computed tomography and three-dimensional printing technology in the restoration of a maxillectomy patient using a dental implant retained obturator. The Journal of Indian Prosthodontic Society 17(4): 406-411.

12. Yeshwanth Pulijala, Ashraf Ayoub (2019) The application of virtual reality and augmented reality in Oral \& Maxillofacial Surgery. BMC Oral Health.

13. Chong Wang, Wei Huang, Yu Zhou, Libing He, Zhi He, et al. (2020) 3D printing of bone tissue engineering scaffolds. Bioactive Materials p. 82 91.

14. Karl Erik Kahnberg (2005) Bone Grafting Techniques for Maxillary Implants. Blackwell Munksgaard pp. 1-3.

15. SJ Froum (2016) Complications of autogenous bone grafting. Dental Implant Complications: Etiology, Prevention, and Treatment, Second Edition. John Wiley \& Sons pp. 332-361.

16. CN Vila (2015) Reconstructive Oral and Maxillofacial surgery. Springer International Publishing, pp. 1-38.

17. Orti Valerie, Frédéric Cuisinier, Jacques Yachouh, Ivan Vladislavov Panayotov (2016) Polyetheretherketone (PEEK) for medical applications. Journal of Material Science 27(7).

18. Maliheh Sadeghi, Izman Sudin, Nida Iqbal, Mohammed Rafiq, Davood Almasi (2016) Preparation Methods for Improving PEEK's Bioactivity for Orthopedic and Dental Application: A Review. International Journal of Biomaterials.

19. (2016) Rehabilitation, Reconstruction, and Regeneration. Biomedical Engineering Society.

20. Yoshiharu Katsumi, Masahide Koyachi, Yu Koyama, Satoru Matsunaga, Keisuke Sugahara (2018) Novel condylar repositioning method for 3Dprinted models. Maxillofacial Plastic and Reconstructive 40(1): 4.

21.D Sarment (2004) Three-Dimensional Planning in Maxillofacial Reconstruction of Large Defects Using Cone Beam Computed Tomography. Cone Beam Computed Tomography Oral and Maxillofacial Diagnosis and Applications, John Wiley \& Sons pp. 109-125. 
ISSN: 2574-1241

DOI: 10.26717/BJSTR.2021.33.005469

Kenan Saoud. Biomed J Sci \& Tech Res

(C) (P) This work is licensed under Creative

Submission Link: https://biomedres.us/submit-manuscript.php

$\begin{array}{ll}\text { BIOMEDICAL } & \text { Assets of Publishing with us } \\ \text { RESEARCHES } & \text { Global archiving of articles } \\ \text { - Immediate, unrestricted online access } & \text { - Rigorous Peer Review Process } \\ \text { https://biomedres.us/ }\end{array}$

